\title{
Elaboração e aplicação de uma tecnologia educacional leve sobre o calendário nacional de imunização infantil no Brasil
}

\author{
Development and application of a lightweight educational technology on the national childhood \\ immunization calendar in Brazil
}

Desarrollo y aplicación de una tecnología educativa ligera en el calendario nacional de vacunación infantil en Brasil

Wesley Brandão Dias

ORCID: https://orcid.org/0000-0001-5033-9844 Universidade do Estado do Pará, Brasil E-mail: wesleybrandao437@gmail.com

Margarete Feio Boulhosa

ORCID: https://orcid.org/0000-0003-1680-9368 Universidade do Estado do Pará, Brasil E-mail: margareteboulhosa@gmail.com

Vinícius Martins Machado ORCID: https://orcid.org/0000-0002-6306-6379 Centro Universitário do Leste de Minas Gerais, Brasil

E-mail: machadoih@yahoo.com.br

Michele de Pinho Barreiros

ORCID: https://orcid.org/0000-0002-7444-6238 Universidade Estadual da Paraíba, Brasil E-mail: mestrandamichele@gmail.com Lindemberg Arruda Barbosa

ORCID: https://orcid.org/0000-0003-2341-5500 Centro Universitário UNIFACISA, Brasil

E-mail: lindemberg.uf@gmail.com

Bruna Jaqueline Ribeiro Lima

ORCID: https://orcid.org/0000-0002-2612-6707 Universidade do Estado do Pará, Brasil

E-mail: jaquelynelima8@gmail.com

Rayane Franklin Mourão Cardoso

ORCID: https://orcid.org/0000-0002-0299-2432 Universidade do Estado do Pará, Brasil E-mail: rayanefranklin20@gmail.com Lis Gomes Rodrigues

ORCID: https://orcid.org/0000-0003-4069-1849 Universidade do Estado do Pará, Brasil

E-mail: lisrodrigues.lr@gmail.com

Lucas Moraes Andrade

ORCID: https://orcid.org/0000-0002-5618-7981 Universidade do Estado do Pará, Brasil E-mail: lucamoraesandrade@gmail.com Wanne Letícia Santos Freitas

ORCID: https://orcid.org/0000-0002-6726-5148 Universidade do Estado do Pará, Brasil E-mail: wanneefreitas@gmail.com

Flávia Savana Ribeiro de Sales ORCID: https://orcid.org/0000-0003-2409-9503 Universidade do Estado do Pará, Brasil E-mail: sssf.sales99@gmail.com

Anna Carolina Rocha de Paiva ORCID: https://orcid.org/0000-0001-6831-8718 Universidade do Estado do Pará, Brasil E-mail: godoikawa4@gmail.com

Pablo Cordovil Lobato dos Santos ORCID: https://orcid.org/0000-0002-8269-3511 Universidade do Estado do Pará, Brasil E-mail: pablolobatto97@gmail.com

Liliane Rogério da Conceição

ORCID: https://orcid.org/0000-0003-2848-4791 


\title{
Resumo
}

O Programa Nacional de Imunização foi criado com o objetivo de promover a saúde populacional. No que concerne ao público infantil, cuja imunização representa um direito inviolável, é fundamental a constante sensibilização dos responsáveis acerca da temática, mitigando a possibilidade do retorno de doenças debeladas. Dessa forma, esse artigo objetivou descrever uma vivência acadêmica ocorrida durante uma ação de educação em saúde, mediante criação e aplicação de uma tecnologia leve voltada aos genitores/responsáveis a respeito da importância da vacinação do público infantil. Percebeu-se no retorno dos participantes que a atividade foi fundamental para a obtenção e consolidação de conhecimentos a respeito da temática abordada. Este estudo possibilitou a compreensão de aspectos referentes a recusa vacinal no Brasil, tendo grande significância aos acadêmicos, uma vez que puderam atuar diretamente nesta realidade.

Palavras-chave: Tecnologia educacional; Vacinação; Promoção da saúde.

\begin{abstract}
The National Immunization Program was created with the aim of promoting population health. With regard to children, whose immunization represents an inviolable right, it is essential to constantly sensitize those responsible about the issue, mitigating the possibility of the return of eradicated diseases. Thus, this article aimed to describe an academic experience that took place during a health education action, through the creation and application of a light technology aimed at parents/guardians regarding the importance of vaccination of children. It was noticed in the participants' feedback that the activity was essential for obtaining and consolidating knowledge about the topic addressed. This study enabled the understanding of aspects related to vaccine refusal in Brazil, having great significance for academics, since they were able to act directly in this reality.
\end{abstract}

Keywords: Educational technology; Vaccination; Health promotion.

\section{Resumen}

El Programa Nacional de Inmunizaciones se creó con el objetivo de promover la salud de la población. Con respecto a los niños, cuya inmunización representa un derecho inviolable, es fundamental sensibilizar constantemente a los responsables sobre el tema, mitigando la posibilidad de que regresen las enfermedades erradicadas. Así, este artículo tuvo como objetivo describir una experiencia académica que tuvo lugar durante una acción de educación en salud, a través de la creación y aplicación de una tecnología ligera dirigida a los padres / tutores sobre la importancia de la vacunación de los niños. En la retroalimentación de los participantes se advirtió que la actividad fue fundamental para la obtención y consolidación de conocimientos sobre el tema abordado. Este estudio permitió comprender aspectos relacionados con el rechazo de vacunas en Brasil, de gran trascendencia para los académicos, ya que pudieron actuar directamente en esta realidad.

Palabras clave: Tecnología educacional; Vacunación; Promoción de la salud.

\section{Introdução}

O Programa Nacional de Imunização (PNI) instituído no Brasil foi criado com o objetivo de promover a saúde populacional, bem como prevenir doenças imunobiologicamente erradicáveis. Dessa forma, todos os segmentos etários possuem necessidades vacinais específicas, devendo ser contempladas através do Sistema Único de Saúde (SUS), mediante os serviços contidos no nível primário de saúde. No que concerne ao público infantil, cuja imunização representa um direito inviolável, é fundamental a constante sensibilização dos responsáveis acerca da temática, mitigando a possibilidade do retorno 
de doenças debeladas (Domingues et al., 2020; Fonseca \& Buenafuente, 2021; Santos, Costa, Silva, Santos \& Gomes, 2017).

A atenção primária caracteriza-se como um ambiente propício para o desenvolvimento de ações educativas, em especial visando a sensibilização do público-alvo sobre uma temática, uma vez que esse nível possui as ferramentas e os recursos necessários para esse fim. Dentre as principais atividades desenvolvidas nesse âmbito, evidenciam-se as ações educativas, as quais têm foco principal na promoção da saúde da população através da conscientização e mudança de hábitos, com estímulo a adoção de um estilo de vida saudável (Giordani, Amaral Júnior, Hugo \& Hilgert, 2020; Siqueira et al., 2017).

Dentre as principais ferramentas utilizadas com finalidade educacional destaca-se o uso das tecnologias educacionais, em especial a leve, cujo conceito perpassa pelas relações sociais e criações de vínculos longitudinais com os usuários do serviço, atuando como mediador das atividades em saúde, subsidiando um cuidado humanizado e respeitoso durante a troca de conhecimentos. Além disso, esse tipo de ferramenta também é conhecida por propiciar a autonomia do indivíduo, incentivando uma autogestão eficaz em saúde (Coelho \& Jorge, 2009; Lisboa, Santos \& Lima, 2017; Lima Neto, Silva \& Santos, 2019).

Ademais, evidencia-se que a atuação dos profissionais da saúde em atividades educativas auxilia na consolidação dos conhecimentos, bem como no estabelecimento de uma relação de confiança com o usuário, fato que permite a abertura para intervenções específicas em saúde (Alves \& Aerts, 2011; Falkenberg et al., 2014; Bezerra et al., 2014). Dessa forma, esse artigo objetivou descrever uma vivência acadêmica ocorrida durante uma ação de educação em saúde, mediante criação e aplicação de uma tecnologia leve (cartilha) voltada à sensibilização dos genitores/responsáveis a respeito da importância da vacinação do público infantil.

\section{Metodologia}

Este estudo trata-se de um estudo descritivo, de cunho qualitativo, do tipo relato de experiência baseado na fundamentação teórica e metodológica da teoria da Problematização a partir da utilização do Arco de Maguerez, o qual é composto por cinco etapas, a saber: 1 - Observação da Realidade; 2 - Levantamento de Pontos-chave; 3 - Teorização; 4 Hipóteses de Solução e 5 - Retorno à Realidade (Berbel, 2011) (Figura 1).

Figura 1: Representação esquemática da operacionalização do arco de Maguerez.

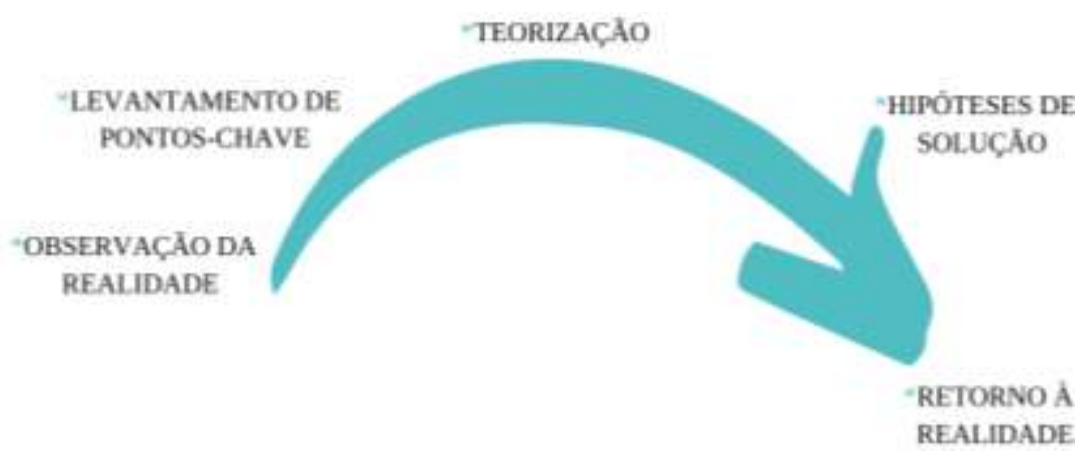

Fonte: Autores (2021).

O local desta pesquisa situa-se em unidade básica de saúde em Belém - PA, destinada ao ensino e assistência com características organizacionais de policlínica, pois desenvolve serviços em vários níveis de complexidade, entre eles, o da atenção básica e de especialidades. Por meio de uma visita in loco, os pesquisadores conheceram os serviços ofertados, bem como todo o seu processo organizacional. Após isso, se levantaram os pontos chave tendo em vista os achados na observação da realidade, a fim de elencar qual seria a intervenção naquela realidade. Por meio dessa discussão, os pesquisadores concluíram que seria de grande valia desenvolver um estudo que envolvesse a participação dos usuários da unidade em uma 
ação de educação em saúde voltada para importância da imunização.

Por conseguinte, a etapa de teorização torna-se um subsídio teórico e científico para o estudo em questão, perpassando pela busca de artigos científicos na Biblioteca Virtual em Saúde (BVS), com a utilização de descritores, obtidos nos Descritores em Ciências da Saúde (DeCS), sendo eles: "Cobertura Vacinal”, "Imunização", "Programas de Imunização" e "Criança". A etapa de hipótese de solução foi definida a partir da convergência das três primeiras etapas da metodologia da Problematização do Arco de Maguerez o que culminou para a idealização de uma ação educativa em saúde sobre o calendário nacional de imunização infantil. Os pesquisadores ainda elaboraram um instrumento educativo no formato de folder para facilitar no processo de transmissão das informações sobre a vacinação infantil. O retorno à realidade ocorreu nas duas primeiras semanas de agosto de 2021.

\section{Resultados}

Dentre os resultados obtidos a partir do Arco de Maguerez, na observação da realidade, os pesquisadores visualizaram o perfil da unidade de maneira abrangente, bem como todo o seu processo organizacional. O serviço que mais chamou a atenção dos pesquisadores foi a sala de vacina, pois a mesma estava vazia, sem filas, e sem expectativa para receber usuários no dia em questão. Este cenário propiciou a discussão para o levantamento de pontos-chave, com isso, os pesquisadores começaram a se perguntar qual fato teria originado tal situação.

A fim de encontrar uma resposta para a indagação, os pesquisadores colheram informações diretamente com a Enfermeira responsável pelo serviço, a mesma relatou que essa situação ainda é um resquício do medo causado pela pandemia Sars-cov-2, medo este em adentrar nas unidades de saúde e se contaminar com o vírus. Outro fator que propicia a baixa adesão no serviço é a crescente veiculação de falsas informações referentes às vacinas disseminadas pelos movimentos antivacina. Ao finalizar seu relato a Enfermeira ressaltou ainda que a procura pela imunização de crianças na unidade estava baixa.

O levantamento dos pontos-chave norteou os pesquisadores a desenvolverem uma atividade referente a vacinação, objetivando conscientizar os usuários da unidade sobre a importância e a necessidade da vacinação, dando ênfase ainda a imunização infantil. Na busca por fundamentação teórica nas bases de dados, os artigos selecionados para este estudo foram: 146 anos do Programa Nacional de Imunizações: uma história repleta de conquistas e desafios a serem superados; 2 Conhecimento, atitude e prática dos vacinadores sobre vacinação infantil em Teresina-PI; 3 - Avaliação da organização e funcionamento das salas de vacina na Atenção Primária à Saúde em Montes Claros, Minas Gerais; 4 - Análise das coberturas vacinais de crianças menores de um ano em Roraima.

A hipótese de solução foi marcada pela elaboração de uma ação educativa em saúde, a fim de intervir positivamente na realidade do local objetivando reverter o quadro referente a falta de adesão a sala de vacinação. Para facilitar o processo de passagem de informações, os participantes elaboraram um folder educativo. O folder intitulado "vacinação infantil em dia" (Figura 2) possui uma linguagem acessível de fácil compreensão direcionada a comunidade. Nele está incluso informações referentes a importância e a necessidade da vacinação, bem como o esquema vacinal preconizado pelo ministério da saúde para o público infantil. 
Research, Society and Development, v. 10, n. 15, e319101522900, 2021

(CC BY 4.0) | ISSN 2525-3409 | DOI: http://dx.doi.org/10.33448/rsd-v10i15.22900

Figura 2: Folder ilustrativo "vacinação infantil em dia”, frente e verso.
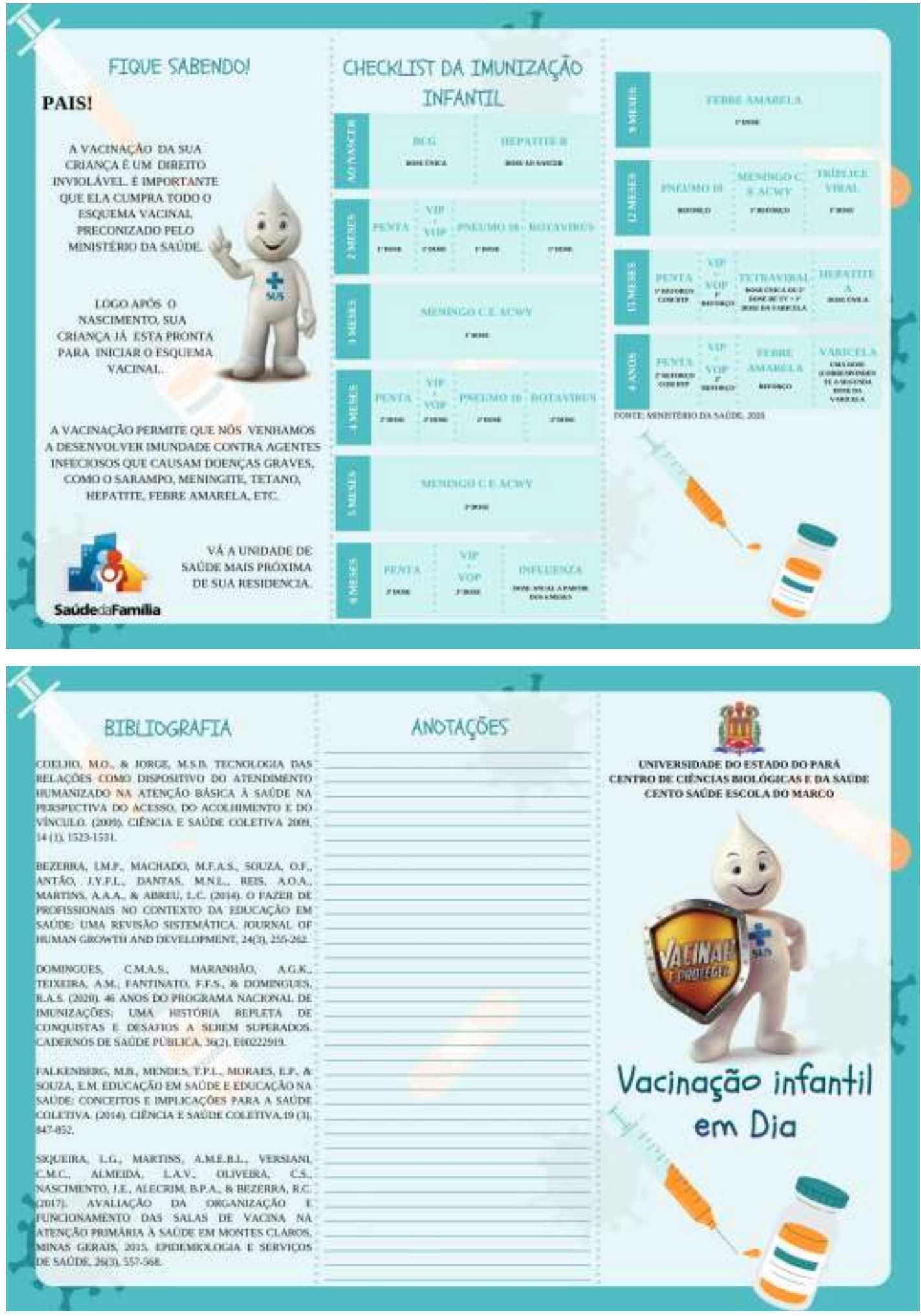

Fonte: Autores (2021).

O retorno à realidade perdurou por duas semanas na unidade de saúde em questão. A operacionalização da educação em saúde contou com a participação de 18 acadêmicos pertencentes a cursos da área da saúde. Ao todo, o grupo acionista abordou para a ação um quantitativo de 63 pessoas. A ação ocorreu da seguinte forma: os acadêmicos abordaram as pessoas que transitavam livremente pelas dependências da unidade convidando-as a participar da ação, informando o tempo de 
duração, que levaria em média de 10 minutos e que nela seria abordada a temática da imunização infantil. A partir do aceite em participar da ação os usuários eram levados para um espaço da unidade, onde os acadêmicos explanaram a respeito da imunização infantil com o auxilio do folder, abrindo espaço para a retirada de dúvidas, após a conversa o participante ganhava o folder, visando a consolidação e a disseminação dos conhecimentos obtidos.

Após a realização e encerramento das ações, os acadêmicos participantes reuniram-se e reportaram sua vivência em discutir sobre as vacinas com os usuários participantes da ação, expondo aquilo que mais chamou sua atenção referente a baixa adesão à vacinação. A maioria dos relatos dos participantes convergiu para a falta de informações sobre a vacinação; algumas pessoas relataram que não tomavam as vacinas por terem medo de adquirir doenças; outras por fobia de agulha; alguns pais ainda justificaram o atraso no calendário vacinal de seus filhos em virtude da contaminação pela pandemia e por não confiarem totalmente na eficácia da vacinação, fatos estes que foram mitigados durante a ação. Dessa forma, notou-se que a ação foi benéfica uma vez que os participantes mostraram-se bastante interessados no que estava sendo ministrado, participando ativamente com indagações e compartilhamento de experiências.

\section{Discussão}

Evidencia-se a partir da ação vivenciada, a importância de ações educativas no nível primário de assistência, objetivando a desmistificação de tabus acerca de diversas temáticas. Percebeu-se no retorno dos participantes que a atividade foi fundamental para a obtenção e consolidação de conhecimentos a respeito da importância da vacinação infantil. De acordo com Kessler et al. (2018), ações de saúde no âmbito da atenção básica são fundamentais para promover a saúde da população, bem como prevenir agravos evitáveis.

Ademais, Cervera, Parreira e Goulart (2011) corroboram o exposto enfatizando que vivências nesse contexto são essenciais para fomentar a adesão aos hábitos adequados de saúde, fato que influencia diretamente na qualidade de vida da população, incentivando uma autogestão eficiente e o aumento da autonomia e criticidade do usuário ante os serviços de saúde. Dessa forma, ainda conforme o autor, essas ações são relevantes no cenário nacional de saúde, uma vez que representam uma intervenção eficaz e simples de ser realizada, porém com reflexos sólidos na vida do público-alvo.

Para Prado e Santos (2018), a execução de medidas educativas junto à população é imprescindível para o estabelecimento e manutenção do vínculo dos profissionais do serviço com os usuários, uma vez que através dessas ferramentas é possível conhecer o público, bem como o nível de instrução de cada um, auxiliando e modelando a forma de ensinar a partir das demandas e necessidades individuais, mitigando, assim, comportamento prejudiciais, bem como fortalecendo a relação de confiança com o usuário do sistema.

Outrossim, evidenciou-se nesse estudo que a utilização da problematização, através do Arco de Maguerez, foi positiva, uma vez que, a partir dela, foi possível identificar previamente as necessidades locais, executando um diagnóstico confiável do contexto a ser trabalhado, bem como elencando as soluções específicas para cada lacuna apresentada. Para Silva et al. (2020), essa metodologia auxilia diretamente na análise situacional local, possibilitando a criação de um planejamento singular a partir das necessidades incialmente identificadas, culminando em uma intervenção condizente com os achados.

Destarte, o uso da tecnologia leve (folder) se fez eficaz para o repasse de conhecimentos, uma vez que propiciou a sensibilização do público-alvo acerca da adesão à vacinação infantil. Assim, conforme evidenciado por Gomes et al. (2021), essas ferramentas são adequadas para o uso com a população, uma vez que facilitam o aprendizado, fazendo com que ele ocorra de maneira horizontalizada, fomentando o saber de maneira humanizada e flexível.

Além disso, Araújo et al. (2020) ainda afirma que essa utilização também é fundamental para o empoderamento do usuário dentro dos serviços de saúde, uma vez que através dela ele pode ser capacitado a respeito dos seus direitos e deveres em saúde, bem como cientificado sobre os hábitos que propiciam melhor qualidade de vida, ratificando a função primordial da 
atenção básica em favorecer a autonomia e a criticidade do usuário, ao passo que o torna sujeito ativo e gestor de sua saúde.

Ademais, Silva et al. (2020) corrobora a importância dessas atividades para a comunidade, haja vista que a partir desse repasse e do estímulo ao conhecimento e debate de temáticas relevantes dentro do cenário assistencial, o usuário, além de obter maiores informações acerca dos assuntos, também serve como propagador entre os membros da comunidade, disseminando os conceitos trabalhados de maneira facilitada, oportunizando a propagação de saberes.

Para mais, ratifica-se também a importância da inserção de acadêmicos da área da saúde em intervenções dentro da comunidade, a partir das quais ele pode vivenciar diferentes realidades, bem como atuar diretamente na solução das problemáticas encontradas. Para Pimenta et al. (2021), esse fato modifica o olhar do profissional, fomentando a efetivação de uma assistência humanizada e integral, culminando em um cuidado diferenciado dentro dos serviços de saúde.

\section{Conclusão}

Este estudo possibilitou a compreensão de alguns aspectos referentes à recusa vacinal no Brasil, tendo significância aos acadêmicos, uma vez que puderam atuar diretamente nesta realidade. Com isso, os pesquisadores alcançaram o objetivo proposto, de maneira exitosa, posto que a partir dele puderam associar a teoria com a resolução de problemas de forma prática, buscando disseminar conhecimentos, bem como desenvolver e aprimorar o seu perfil profissional através da elaboração e aplicação da tecnologia sobre o calendário nacional de imunização. Estudos como este mostram-se necessários, pois estreitam os laços entre os profissionais e os usuários do serviço em questão e ainda auxiliam diretamente no aperfeiçoamento do serviço de acordo com as suas necessidades específicas. Sendo assim, a unidade de saúde é beneficiada, tendo em vista que os seus serviços estarão sendo ofertados de forma clara, suprindo a demanda da população local. Sugere-se para estudos futuros a aplicação de tecnologias educativas em múltiplos locais, em especial dentro dos serviços da atenção básica, visando maior variabilidade de resultados e propagação de informações em saúde dentro da comunidade.

\section{Referências}

Alves, G. G., \& Aerts, D. As práticas educativas em saúde e a Estratégia Saúde da Família. (2011). Ciência e Saúde Coletiva, 16 (1), $319-325$.

Araujo, L. T., Silva, J. M. L., Santos, S. R. P., Santos, P. C. L., Conceição, L. R., Cunha, A. G., Lopes, L. S., Saldanha, I. C. S., Ferreira, L. F., Souza, G. O., Anjos, R. B., Soares, M. K. M., Souza, L. R., Cardoso, M. E. O., \& Lima, C. B. M. (2020). Educational actions in high-risk prenatal care for pregnant women with heart disease: strategies for efficient self-care. Research, Society and Development, 9(10), e9789109405.

Bezerra, I. M. P., Machado, M. F. A. S., Souza, O. F., Antão, J. Y. F. L., Dantas, M. N. L., Reis, A. O. A., Martins, A. A. A., \& Abreu, L. C. (2014). O fazer de profissionais no contexto da educação em saúde: uma revisão sistemática. Journal of Human Growth and Development, $24(3), 255-262$.

Berbel, N. A. N. (2011). As metodologias ativas e a promoção da autonomia de estudantes. Semina: Ciências Sociais e Humanas, 32(1), 25-40.

Cervera, D. P. P., Parreira, B. D. M., \& Goulart, B. F. Educação em saúde: percepção dos enfermeiros da atenção básica em Uberaba (MG). (2011). Ciência e Saúde Coletiva, 16 (1), 1547-1554.

Coelho, M.O., \& Jorge, M. S. B. Tecnologia das relações como dispositivo do atendimento humanizado na atenção básica à saúde na perspectiva do acesso, do acolhimento e do vínculo. (2009). Ciência e Saúde Coletiva 2009, 14 (1), 1523-1531.

Domingues, C. M. A. S., Maranhão, A. G. K., Teixeira, A. M., Fantinato, F. F. S., \& Domingues, R. A. S. (2020). 46 anos do Programa Nacional de Imunizações: uma história repleta de conquistas e desafios a serem superados. Cadernos de Saúde Pública, 36(2), e00222919.

Falkenberg, M. B., Mendes, T. P. L., Moraes, E. P., \& Souza, E. M. Educação em saúde e educação na saúde: conceitos e implicações para a saúde coletiva. (2014). Ciência e Saúde Coletiva,19 (3), 847-852.

Fonseca, K. R., \& Buenafuente, S. M. F. (2021). Análise das coberturas vacinais de crianças menores de um ano em Roraima, 2013-2017. Epidemiologia e Serviços de Saúde, 30(2), e2020195.

Giordani, J. M. A., Amaral Júnior, O. L., Hugo, F. N., \& Hilgert, J. B. (2020). Fatores associados à realização de acolhimento pelas equipes da Atenção Básica à Saúde no Brasil, 2012: um estudo transversal. Epidemiologia e Serviços de Saúde, 29(5), e2019468.

Gomes, A. Q., Paiva, A. C. R., Cardoso, C. C., Azevedo, C. M., Baia, D. F. S., Tavares, I. I. S., Pereira, I. J., Silva, J. M. L., Barros, K. B. C., Lopes, L. S., Sarges, M. R. V., Barreiros, M. P., Soares, M. K. M., Silva, P. L. C., Franco, T. C., \& Costa, T. S. (2021). Leprosy in primary health care: Educational activities in health and its preventive role. Research, Society and Development, 10 (7), e26610715702. 
Research, Society and Development, v. 10, n. 15, e319101522900, 2021

(CC BY 4.0) | ISSN 2525-3409 | DOI: http://dx.doi.org/10.33448/rsd-v10i15.22900

Kessler, M., Thumé, E., Duro, S. M. S., Tomasi, E., Siqueira, F. C. V., Silveira, D. S., Nunes, B. P., Volz, P. M., Santos, A. A., França, S. M., Bender, J. D., Piccinini, T., \& Facchini, L.A. (2018). Ações educativas e de promoção da saúde em equipes do Programa Nacional de Melhoria do Acesso e da Qualidade da Atenção Básica, Rio Grande do Sul, Brasil. Epidemiologia e Serviços de Saúde, 27 (2), e2017389.

Lima Neto, A. V., Silva, M. F., \& Santos, V. E. P. (2019). Contribuições das tecnologias em saúde para a segurança do paciente. Revista Cubana de Enfermería, 35(3), e2125.

Lisboa, N. A., Santos, S. F., \& Lima, E. I. (2017). A importância das tecnologias leves no processo de cuidar na atenção primária em saúde. Textura, 10(19), $164-171$.

Pimenta, A. G. D., Mendes, A. P. S., Braga, B. S. C., Silva, B. V. C., Teixeira, D., Ramos, E. C., Gomes, E. S., Pantoja, G. X., Pinto, I. M., Nascimento, J. L. M., Silva, J. M. L., Costa, J. R. M., Souza, L. R., Silva, L. M., Araujo, L. T., Ferreira, L. G., Soares, M. K. M., \& Barros, V. V. (2021).Health education as a tool in the prevention of childhood pneumonia: a case report. Research, Society and Development, 10(2), e13410212039.

Prado, N. M. B. L., \& Santos, A. M. Promoção da saúde na Atenção Primária à Saúde: sistematização de desafios e estratégias intersetoriais. (2018). Saúde em Debate, 42(1), 379-395.

Santos, C. A. P. S., Costa, R. S., Silva, J. L. M., Santos, M. R. F., \& Gomes, B. L. F. (2017). Conhecimento, atitude e prática dos vacinadores sobre vacinação infantil em Teresina-PI, 2015. Epidemiologia e Serviços de Saúde, 26(1), 133-140.

Silva, J. M. L., Leal, A. C., Anjos, R. B., Cunha, A. G., Rocha, S. G., Lima, C. B. M., Tavares, I. I. S., Matos, A. L. A., Anjos, J. P. N., Matos, D. C., Couto, S.S.T., Silva, L. C., Campos, M. I., Santos, A. C. S, \& Costa, J. R.M. (2020). Prostate cancer in primary care: mitigation strategies through health education. Research, Society and Development, 9 (11), e3049119829.

Silva, J. M. L., Monteiro, A. J. C., Coutinho, E. S., Cruz, L. B. S., Araujo, L. T., Dias, W. B., \& Costa, P. V. D. P. (2020). The instructional therapeutic toy as a tool in child cancer care. Research, Society and Development, 9 (7), e408974253.

Siqueira, L. G., Martins, A. M. E. B. L., Versiani, C. M. C., Almeida, L. A. V., Oliveira, C. S., Nascimento, J. E., Alecrim, B. P. A., \& Bezerra, R. C. (2017). Avaliação da organização e funcionamento das salas de vacina na Atenção Primária à Saúde em Montes Claros, Minas Gerais, 2015. Epidemiologia e Serviços de Saúde, 26(3), 557-568 